\title{
Adoção de Blended Learning: verificação do potencial de ampliação na Universidade Federal do Vale do São Francisco
}

\author{
Jorge Luis Cavalcanti Ramos ${ }^{1}$, Ramona Florêncio Pereira da Silva ${ }^{1}$, João Carlos \\ Sedraz Silva ${ }^{1}$, Alex Sandro Gomes ${ }^{2}$. \\ Universidade Federal do Vale do São Francisco (UNIVASF) ${ }^{1}$ \\ Universidade Federal de Pernambuco (UFPE) ${ }^{2}$ \\ jorge.cavalcanti@univasf.edu.br, joao.sedraz@univasf.edu.br, \\ ramonaflorenciol07@hotmail.com, asg@cin.ufpe.br
}

\begin{abstract}
This paper presents a study that aims to assess the current level of adoption of virtual learning environment Moodle to support classroom learning, featuring Blended Learning at the Federal University of Vale do São Francisco (UNIVASF). A detailed survey of the usage level of the main features of the virtual environment by teachers and the percentage of use by many of the courses and subjects was done to verify the potential for growth of the modality. The results show that there is great potential for growth of Blended Learning in the institution and the need to establish strategy to support and encourage this growth.
\end{abstract}

Resumo. Este artigo apresenta um estudo que teve como objetivo avaliar o nível atual de adoção do ambiente virtual de aprendizagem Moodle no apoio ao ensino presencial, caracterizando o Blended Learning na Universidade Federal do Vale do São Francisco (UNIVASF). Foi feito um levantamento detalhado do nivel de uso das principais funcionalidades do ambiente virtual pelos professores e o percentual de utilização do mesmo pelos diversos cursos e disciplinas, para verificar o potencial de incremento da modalidade. Os resultados mostram que existe um grande potencial para crescimento do Blended Learning na instituição e a necessidade de se estabelecer estratégia para apoiar e estimular esse crescimento.

\section{Introdução}

Os Ambientes Virtuais de Aprendizagem (AVAs) são, de modo simplificado, um espaço ou conjunto de elementos tecnológicos que usam a internet como plataforma operacional e que tem como principal objetivo facilitar a transmissão de diferentes tipos de conhecimento seja de modo síncrono ou assíncrono, através de suas atividades como: chat, fórum, tarefa etc. $\mathrm{O}$ uso desses ambientes pode ser estendido a diversas plataformas e dispositivos, tais como tablets e smartphones, conforme descrito em Voss et al. (2013),

Os Ambientes Virtuais de Aprendizagem trazem novas perspectivas para o processo de ensino-aprendizagem, o que requer dos docentes um olhar inovador, não apenas na forma como se entende a educação, mas principalmente na forma como ocorre a prática, garantindo uma verdadeira mudança de paradigma nesta modalidade educativa, indo além do tradicionalismo a que se encontra interligada (Araújo e Panerai 2012).

Uma das modalidades de ensino baseado em AVAs é o Blended Learning, ou simplesmente, ensino híbrido/misto (Tori, 2009), onde são combinadas as características do ensino presencial com o ensino a distância mediado por computador (e-learning) (Rodrigues, 2010). Uma das suas principais características é a versatilidade de utilizar 
encontros presenciais da maneira tradicional, como também disponibilizar material didático e realizar atividades online, por exemplo, postagens de links, utilizar fóruns, entregas controladas de tarefas dentre outros (Silva et al., 2013).

Diante desse contexto, tal modelo vem sido adotado por vários professores da Universidade Federal do Vale do São Francisco (UNIVASF) que utilizam o Moodle $e^{l}$ como plataforma de apoio às suas disciplinas presenciais. Embora o ambiente tenha vários recursos, estima-se que a maioria dos professores utiliza apenas aqueles mais básicos, como disponibilização de material, fóruns e envio de tarefas controladas por data.

Conforme levantamento feito por Gluz et al. (2011), naquele ano, cerca de 98\% das instituições de ensino públicas no país usavam o Moodle, sendo que algumas usavam mais de um ambiente. $\mathrm{O}$ estudo não precisou a distribuição do uso do ambiente nas modalidades a distância, presencial ou híbrida.

Este estudo teve como objetivo analisar o nível de utilização do AVA no Blended Learning nos diversos cursos da UNIVASF, avaliando assim o possível incremento da sua adoção, a partir da sugestão de ações para potencializar essa utilização do ambiente virtual no apoio às disciplinas presenciais.

Este trabalho está estruturado da seguinte forma: a seção 2 descreve o embasamento teórico deste estudo. $\mathrm{Na}$ seção 3 são apresentados os procedimentos metodológicos da pesquisa. Os resultados e suas discussões são apresentados na seção 4. Finalmente, na seção 5 são feitas as considerações finais e sugestões de trabalhos futuros para continuidade desta pesquisa.

\section{Fundamentação Teórica}

A ideia de Blended Learning $(\boldsymbol{B L})$, uma combinação do tradicional ensino face-a-face com instrução mediada por tecnologia, não é uma novidade e está presente no Brasil desde os projetos de educação a distância em meados da década de 1970 (Castro, 2007 apud Rodrigues, 2010). Porém, só veio ter destaque com o avanço tecnológico e a criação de ambientes especializados em tal modalidade, os Ambientes Virtuais de Aprendizagem. Acompanhando tal desenvolvimento, as instituições de ensino superior (IES) tem visto essa modalidade como um processo a agregar valor na formação profissional dos seus alunos, adotando não apenas a modalidade a distância, como também esse modelo híbrido.

\subsection{Blended Learning como abordagem nos processos de ensino-aprendizagem}

A $B L$ é uma abordagem de ensino que possibilita a interação entre as modalidades presencial e não presencial, com a interação entre as abordagens pedagógicas e também a interação entre os recursos tecnológicos utilizados (Cícero, 2012).

A $B L$ pode proporcionar benefícios pedagógicos, tais como o aumento da eficácia da aprendizagem, satisfação e eficiência (Graham, 2013). A BL também demonstra potencial para aumentar o acesso e a flexibilidade dos cursos (Moskal et al., 2013), oferecendo também para professores e alunos uma maior flexibilidade temporal e geográfica, o que lhes permite determinar quando e onde os segmentos online de instrução podem ocorrer (King \& Arnold, 2012).

Ross \& Gage (2006) afirmaram que o uso de $B L$ da mesma forma que diminui o tempo total de atividades presenciais dos cursos, proporciona uma retenção maior do que cursos totalmente online, podendo impactar na redução do tempo para conclusão dos mesmos.

\footnotetext{
${ }^{1}$ www.moodle.univasf.edu.br
} 
Para Rodrigues (2010), a $B L$ não se define apenas à combinação do ensino presencial ao ensino a distância; esse conceito ainda pode congregar uma variedade de recursos tecnológicos e também a combinação de diferentes métodos de ensinoaprendizagem.

$\mathrm{Na}$ prática da $B L$, para se utilizar as novas tecnologias da informação e da comunicação no ensino, são necessários, além do computador, dos softwares e da internet, professores com domínio pedagógico e capazes de refletir sobre as formas adequadas de utilização dos recursos como meio educacional (Valente, 2005).

Outro aspecto a ser observado é que na prática da $B L$ a produção coletiva de conteúdo afasta o discente da posição de figurante, na medida em que o torna protagonista e coautor do material didático (Silva et al., 2013).

\subsection{Estratégia para adoção da $B L$ em Instituições de Ensino Superior}

A adoção de $B L$ é cada vez maior no ensino superior em todo o mundo (Graham et al., 2013). Pesquisadores previram que a $B L$ iria se tornar o "novo modelo tradicional" (Ross \& Gage, 2006), ou o "novo normal" nos cursos superiores (Norberg et al., 2011). No entanto, são escassos os dados disponíveis que indiquem com precisão até que ponto a $B L$ foi realmente adotada no ensino superior, em parte devido a um desacordo sobre como as instituições devem definir, implantar e avaliar a $B L$ (Graham, 2013).

No entanto, muitas IES têm cursos com $B L$ porque foi experimentada ou adotada por iniciativa dos professores, embora a própria instituição não adotasse a modelo oficialmente. A $B L$ começou em muitos lugares como um esforço da base, adotada pelo corpo docente interessado em combinar estratégias online e tradicionais para melhorar os resultados de aprendizagem dos alunos (Graham et al. 2013).

Enquanto muitos estudos têm investigado a eficácia da $B L$ no nível da disciplina individual, muito poucos estudos fornecem orientação para as instituições (Halverson et al., 2012). Em Graham et al. (2013) é feita uma análise da adoção da $B L$ em seis instituições de ensino superior, para orientar gestores na implantação da modalidade. Os casos analisados são usados para identificar e elaborar ações sobre questões fundamentais relacionadas com a estratégia da institucionalização da $B L$. Também nesse trabalho são detalhados os estágios para adoção (Quadro 01) e as categorias para implementação da $B L$ (Quadro 02).

Quadro 01. Resumo das etapas de adoção da BL (Graham et al., 2013).

\begin{tabular}{|l|l|}
\hline \multicolumn{1}{|c|}{ Etapa } & \multicolumn{1}{c|}{ Descrição } \\
\hline $\begin{array}{l}\text { Fase 1: Sensibilização / } \\
\text { exploração }\end{array}$ & $\begin{array}{l}\text { Sensibilização institucional inicial e suporte limitado para possibilitar } \\
\text { cada professor explorar formas em que eles podem empregar técnicas } \\
\text { de } B L \text { em suas aulas. }\end{array}$ \\
\hline $\begin{array}{l}\text { Fase 2: Adoção/ } \\
\text { implementação inicial }\end{array}$ & $\begin{array}{l}\text { Adoção institucional da estratégia de } B L \text { e experimentação de novas } \\
\text { políticas e práticas para apoiar a continuidade de sua implementação. }\end{array}$ \\
\hline $\begin{array}{l}\text { Fase 3: Implementação } \\
\text { madura / crescimento }\end{array}$ & $\begin{array}{l}\text { Estratégias bem estabelecidas para a } B L \text {, onde a estrutura e apoio } \\
\text { agora são partes integrantes das operações gerais da universidade. }\end{array}$ \\
\hline
\end{tabular}

Quadro 02. Resumo das categorias para implantação da BL (Graham et al., 2013).

\begin{tabular}{|l|l|}
\hline \multicolumn{1}{|c|}{ Tema } & \multicolumn{1}{c|}{ Descrição } \\
\hline Estratégia & $\begin{array}{l}\text { Aborda questões relacionadas com o design geral do processo de implantação da } B L, \\
\text { como a definição, formas de divulgação, o grau de implementação e as políticas } \\
\text { institucionais. }\end{array}$ \\
\hline Estrutura & $\begin{array}{l}\text { Aborda questões relativas à estrutura tecnológica, pedagógica, administrativa e } \\
\text { facilitar o ambiente de } B L, \text { incluindo governança, modelos e avaliação. }\end{array}$ \\
\hline Apoio & Aborda questões relacionadas com a maneira pela qual uma instituição facilita a \\
\hline
\end{tabular}




\begin{tabular}{|c|l|}
\hline Tema & \multicolumn{1}{c|}{ Descrição } \\
\hline & $\begin{array}{l}\text { implementação e manutenção de seu projeto } B L, \text { inclusive com apoio técnico, apoio } \\
\text { pedagógico, corpo docente e oportunidades. }\end{array}$ \\
\hline
\end{tabular}

Niemiec e Otte (2009) discutiram a necessidade de determinar claramente como a $B L$ ajuda a instituição cumprir a sua missão e seus objetivos, fornecendo vários exemplos de como uma instituição pode usar a modalidade de maneira objetiva e produtiva.

\section{Procedimentos Metodológicos}

Este estudo foi centrado na análise do número de acessos, recursos disponibilizados e atividades propostas nas diversas disciplinas que usam o Moodle como plataforma de apoio ao ensino presencial na UNIVASF. Dessa forma se buscou um levantamento atualizado do uso das funcionalidades e também o quanto os diversos cursos presenciais utilizam o BL. Os cursos e disciplinas ofertados na modalidade a distância não foram analisados nesta pesquisa.

Para realização desta pesquisa, foi usada a metodologia exploratória de textos relacionados com o tema do trabalho e da análise de dados coletados em um levantamento descrito na seção 3.3 .

\subsection{Objetivo}

Avaliar o nível atual de adoção do BL na instituição a partir da análise do uso Moodle, detalhando a utilização dos seus recursos e atividades e seus respectivos acessos, para verificar o potencial de incremento do uso do Blended Learning na UNIVASF.

\subsection{Contexto da pesquisa}

O ambiente Moodle na UNIVASF possui várias instâncias instaladas, uma para cada propósito: EAD Graduação, EAD Pós-Graduação, EAD Extensão e a de Apoio ao Ensino Presencial. Todas são administradas pela Secretaria de Educação a Distância (SEAD) da instituição. Como este trabalho focou exclusivamente no uso do ambiente no apoio ao ensino presencial, a coleta de dados ocorreu nessa instância do Moodle.

\subsection{Procedimentos para coleta dos dados}

Para a realização da coleta foi necessário o acesso à plataforma com um usuário com perfil de administrador do sistema. Por meio desse login foi gerado o relatório de acessos realizados em cada uma das disciplinas ativas no segundo semestre de 2013. A intenção foi, a partir da identificação das disciplinas com maior número de acessos, estabelecer um ponto de corte para a criação da lista com as turmas que seriam avaliadas nesta pesquisa.

Foram usados como período para geração do relatório de acessos os dois últimos meses do semestre letivo, onde os acessos em todo o ambiente tornam-se mais frequentes, conforme também constatado pelos autores ao comparar esse período com os acessos em outros períodos do semestre.

O relatório do Moodle fornece um ranking com no máximo noventa e nove disciplinas disponibilizadas. Porém, como este relatório apresentou algumas disciplinas com número muito baixo de acessos às atividades, foi levado em consideração como "ativa" aquela disciplina que obteve um mínimo de duzentos acessos no período pesquisado. Este valor equivale a cerca de cem acessos por mês, o que dividido por um número médio de vinte alunos (como em disciplinas específicas, tais como Banco de Dados ou Materiais de Construção, dentre outros) resultaria em apenas cinco acessos de cada aluno no período de quatro semanas. 
A partir desse critério foi possível relacionar as setenta e duas disciplinas mais ativas no ambiente, com as respectivas quantidades de acessos, usuários registrados, professores e curso a qual estava vinculado à disciplina listada.

As principais funcionalidades didáticas do Moodle são agrupadas em duas categorias: recursos e atividades. Entre os recursos, podemos citar "criar página de texto simples", "link a um arquivo ou a página web" e "visualizar diretório". Entre as atividades encontramos "chat", "fórum", "envio de arquivo", "wiki" dentre outras.

Após o levantamento inicial, a coleta de dados foi direcionada para cada uma das setenta e duas disciplinas listadas. Ainda com acesso com o perfil de administrador, a tarefa era entrar na página de cada uma e fazer a verificação da utilização de cada um dos recursos ou atividades do ambiente na disciplina. Essa verificação era registrada em uma planilha eletrônica conforme a Figura 01, onde a presença do recurso ou atividade era colocado o número identificador 1 na respectiva coluna. A partir desse identificador foi possível contar e estabelecer um percentual de frequência de cada funcionalidade por disciplina.

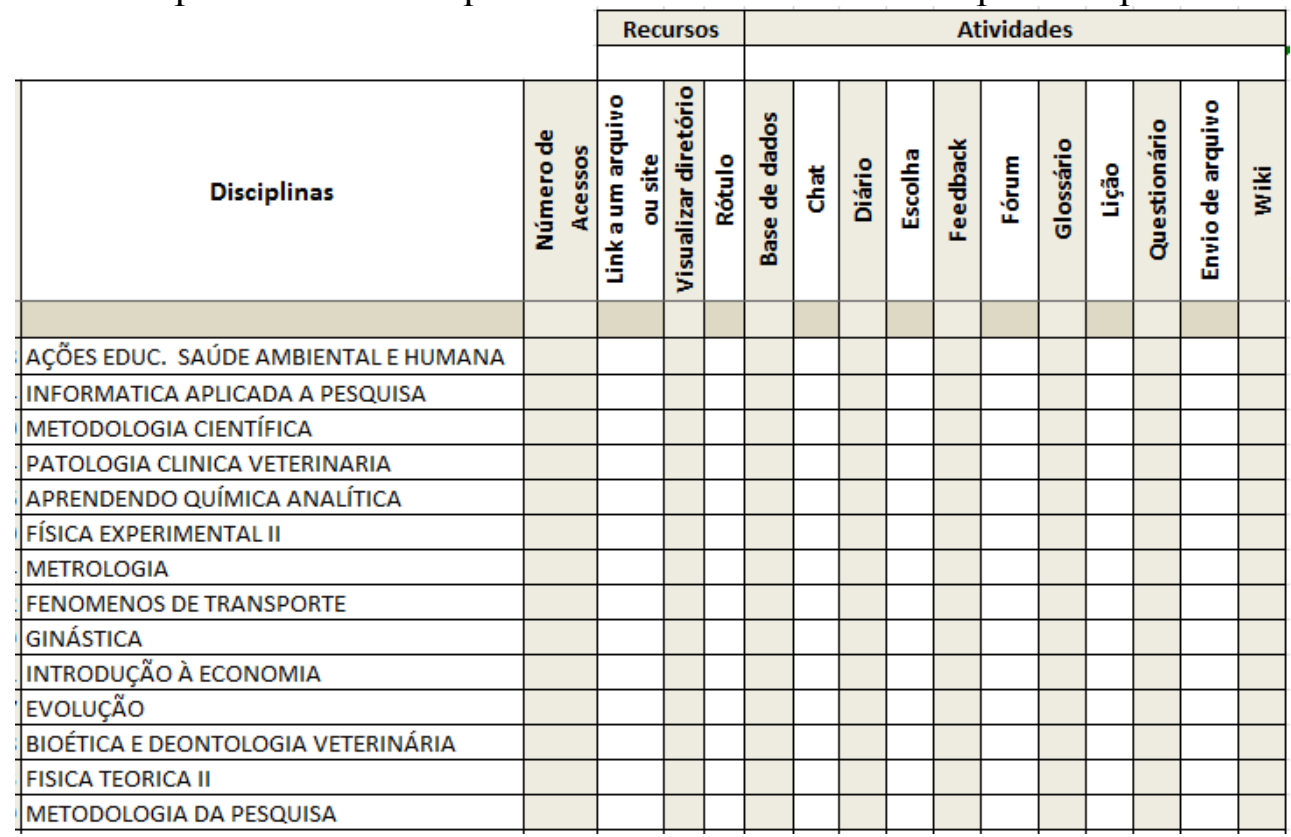

\section{Figura 01. Exemplo da planilha de coleta e registro dos recursos e atividades em cada disciplina.}

De modo análogo, foi usada outra planilha similar com o objetivo de se registrar quantas vezes cada funcionalidade ocorria em cada uma das disciplinas. Assim teríamos outro dado importante que indicaria, entre as funcionalidades mais usadas, quanto cada uma estava sendo usada em cada disciplina.

Essa planilha permitiu a verificação em números percentuais de uso para cada funcionalidade e assim também estabelecer outro ranking de recursos e atividades para permitir, por exemplo, investigar as razões das funcionalidades com baixa utilização.

Outra importante coleta neste experimento foi feita para se saber o número de disciplinas de cada curso de graduação da UNIVASF, por semestre letivo. A intenção era estabelecer uma relação entre o número de disciplinas ativas do curso no Moodle e o número total de disciplinas daquele curso no semestre. Essa relação indicaria quais os cursos de graduação que possuem mais disciplinas usando o Blended Learning e qual a média geral de utilização entre os cursos, para se determinar o quanto esta modalidade ainda pode crescer internamente na IES. 


\section{Resultados e Discussões}

Após a coleta e conferência dos dados nas planilhas, foram feitas algumas análises para obter os resultados deste estudo e possibilitar discussões de estratégias e propostas sobre o Blended Learning na UNIVASF.

\subsection{Análise do levantamento das funcionalidades usadas.}

Em relação às funcionalidades do Moodle que estão presentes nas diversas disciplinas e seus respectivos percentuais de utilização, a Tabela 01 exibe a consolidação dos dados coletados na planilha mostrada na Figura 01, agrupando recursos e atividades presentes nas disciplinas analisadas.

Tabela 01. Ranking das funcionalidades presentes dos diversos cursos no ambiente.

\begin{tabular}{l|c}
\hline \multicolumn{1}{c|}{ Funcionalidades } & Frequência \\
\hline Fórum & $96 \%$ \\
\hline Link a um arquivo/site & $90 \%$ \\
\hline Envio de Arquivos & $51 \%$ \\
\hline Rótulo & $50 \%$ \\
\hline Visualizar diretório & $35 \%$ \\
\hline Questionário & $17 \%$ \\
\hline Chat & $14 \%$ \\
\hline Escolha & $8 \%$ \\
\hline Wiki & $6 \%$ \\
\hline Diário & $6 \%$ \\
\hline Lição & $4 \%$ \\
\hline Glossário & $4 \%$ \\
\hline Feedback & $1 \%$ \\
\hline
\end{tabular}

Esse ranking indica que a atividade "Fórum" está presente em $96 \%$ das disciplinas pesquisadas. Já o recurso "Link a um arquivo/ site", é usado em 90\% das disciplinas. Ao considerarmos um indicador mediano, percebe-se então a preferência pelo uso de somente quatro das funcionalidades do Moodle nessas disciplinas.

A Tabela 02 consolida os dados coletados na planilha da contagem de recursos e atividades por disciplina. Nela é feito o detalhamento a partir da contagem de quantas vezes cada funcionalidade é usada em cada disciplina, depois transformada em percentual, a partir da divisão do número de ocorrências da funcionalidade na disciplina pelo número total de recursos contabilizados em todo o levantamento.

Tabela 02. Ranking das funcionalidades utilizadas do Moodle na UNIVASF.

\begin{tabular}{l|c}
\hline \multicolumn{1}{c|}{ Funcionalidades } & Frequência \\
\hline Link a um arquivo/site & $90 \%$ \\
\hline Envio de Arquivos & $46 \%$ \\
\hline Fórum & $34 \%$ \\
\hline Questionário & $9 \%$ \\
\hline Rótulo & $6 \%$ \\
\hline Visualizar diretório & $3 \%$ \\
\hline
\end{tabular}


Tabela 02. (Continuação)

\begin{tabular}{l|c}
\hline \multicolumn{1}{c|}{ Funcionalidades } & Frequência \\
\hline Lição & $3 \%$ \\
\hline Chat & $2 \%$ \\
\hline Escolha & $2 \%$ \\
\hline Wiki & $1 \%$ \\
\hline Diário & $1 \%$ \\
\hline Glossário & $1 \%$ \\
\hline
\end{tabular}

Para exemplificar os cálculos dos percentuais em ambas as tabelas, tomemos por base a soma dos totais de recursos e a soma dos totais de atividades em todas as disciplinas analisadas, para calcularmos o percentual do Recurso "Link a um arquivo/site" e o percentual para a atividade "Envio de Arquivos", conforme Tabela 03.

\section{Tabela 03. Demonstrativo do cálculo do percentual de recursos e atividades nas} disciplinas analisadas.

\begin{tabular}{l|c}
\hline \multicolumn{1}{c|}{ Indicador } & Total de Ocorrências \\
\hline $\begin{array}{l}\text { Contagem dos recursos disponibilizados } \\
\text { em todas as disciplinas }\end{array}$ & 1692 \\
\hline $\begin{array}{l}\text { Contagem do recurso "Link a um } \\
\text { arquivo/site" nas disciplinas }\end{array}$ & 1530 \\
\hline $\begin{array}{l}\text { \% de utilização do Recurso "Link } \boldsymbol{a} \text { um } \\
\text { arquivo/site" }\end{array}$ & $1530 / 1692=0,904=\mathbf{9 0 \%}$ \\
\hline $\begin{array}{l}\text { Contagem atividades } \\
\text { disponibilizadas em todas as disciplinas }\end{array}$ & 2306 \\
\hline $\begin{array}{l}\text { Contagem da atividade "Envio de } \\
\text { Arquivos" nas disciplinas }\end{array}$ & $230 / 496=0,463=\mathbf{4 6 \%}$ \\
\hline \begin{tabular}{l} 
Arquivos" utilização da Atividade "Envio de \\
\hline
\end{tabular}
\end{tabular}

Os resultados da Tabela 02 indicam a predominância do uso de somente três funcionalidades do Moodle nas diversas disciplinas que o utilizam como apoio ao ensinoaprendizagem. Isso evidencia uma subutilização do ambiente, muito provavelmente por desconhecimento das demais funcionalidades pelos professores usuários ou pela sua opção de não usar outros recursos além dos mais comuns. Isso poderá ser avaliado numa provável continuação deste estudo.

De certa forma, podemos identificar um custo maior de infraestrutura computacional usar somente poucas funções de uma plataforma tão abrangente e que exige bons servidores e demais recursos de rede, pessoal qualificado para suporte, dentre outros requisitos para adoção do Moodle no ambiente acadêmico.

Além da necessidade de capacitação dos professores para uso dos demais recursos e atividades do ambiente, sugere-se ainda a formação de equipe de apoio para design instrucional, que seria responsável pela produção de conteúdo de modo a tornar os materiais didáticos mais dinâmicos e atrativos para os alunos, para estimular a aprendizagem colaborativa e outras ações interessantes para o processo de ensino-aprendizagem.

\subsection{Análise da utilização da modalidade nos diversos cursos e disciplinas.}

Outra etapa da coleta de dados foi o levantamento de quantas disciplinas cada curso de graduação da UNIVASF oferta em cada semestre, já que existem cursos com entradas semestrais e outros com entradas anuais. A intenção é estabelecer um comparativo entre as disciplinas ofertadas e as que estão no ambiente virtual, ativas ou simplesmente criadas, mas 
sem acessos recentes, por não estarem sendo ofertadas no semestre analisado ou por opção do professor em não ativá-la.

$\mathrm{Na}$ Tabela 04 são apresentados os resultados desse levantamento por curso. Observa-se que todos os 21 cursos de graduação da universidade possuem disciplinas criadas no Moodle, mas em pelo menos um terço deles a utilização ainda não é efetiva ou é mínima. Nos cursos que usam o ambiente, ainda assim há uma baixa quantidade de disciplinas criadas e ativas em relação ao total de disciplinas. Apenas quatro cursos tem sua utilização igual ou superior a $20 \%$ do total das disciplinas.

Há também uma discrepância entre o número de disciplinas criadas e o número de disciplinas efetivamente ativas. O fator semestral, onde a disciplina só é ofertada uma vez por ano letivo, pode justificar sua inatividade no semestre avaliado. Mas outros fatores podem influenciar essa inatividade e serão investigados na continuação deste estudo.

Tabela 04. Utilização do Moodle por curso.

\begin{tabular}{|c|c|c|c|c|c|}
\hline Curso & $\begin{array}{c}\text { NDS }(*) \\
\text { Par }\end{array}$ & $\begin{array}{c}\text { NDS } \\
\text { Ímpar }\end{array}$ & $\begin{array}{c}\text { Disciplinas } \\
\text { Criadas }\end{array}$ & $\begin{array}{c}\text { Disciplinas } \\
\text { Ativas no } \\
\text { Semestre } \\
\end{array}$ & Utilização \\
\hline Educação Física & 26 & 26 & 30 & 11 & $42,3 \%$ \\
\hline Eng. Mecânica & 29 & 31 & 19 & 6 & $20,7 \%$ \\
\hline Artes Visuais & 15 & 17 & 16 & 3 & $20,0 \%$ \\
\hline Enfermagem & 10 & 12 & 9 & 2 & $20,0 \%$ \\
\hline Administração & 48 & 48 & 30 & 8 & $16,7 \%$ \\
\hline Eng. de Produção & 33 & 31 & 24 & 5 & $15,2 \%$ \\
\hline Eng. de Computação & 34 & 33 & 22 & 5 & $14,7 \%$ \\
\hline Eng. Agrícola e Ambiental & 35 & 33 & 40 & 5 & $14,3 \%$ \\
\hline Ciências Farmacêuticas & 51 & 51 & 31 & 7 & $13,7 \%$ \\
\hline Medicina Veterinária & 57 & 57 & 9 & 5 & $8,8 \%$ \\
\hline Eng. Civil & 35 & 38 & 26 & 3 & $8,6 \%$ \\
\hline Ciências Biológicas & 48 & 48 & 21 & 4 & $8,3 \%$ \\
\hline Ciências da Natureza SBF & 37 & 37 & 17 & 3 & $8,1 \%$ \\
\hline Eng. Elétrica & 37 & 32 & 16 & 3 & $8,1 \%$ \\
\hline Ciências da Natureza SRN & 14 & 23 & 28 & 1 & $7,1 \%$ \\
\hline Eng. Agronômica & 34 & 34 & 14 & 1 & $2,9 \%$ \\
\hline Arqueologia e Preservação Patrimonial & 20 & 19 & 15 & 0 & $0,0 \%$ \\
\hline Ciências Sociais & 31 & 31 & 2 & 0 & $0,0 \%$ \\
\hline Medicina & 54 & 54 & 15 & 0 & $0,0 \%$ \\
\hline Psicologia & 56 & 56 & 5 & 0 & $0,0 \%$ \\
\hline Zootecnia & 31 & 40 & 12 & 0 & $0,0 \%$ \\
\hline \multicolumn{3}{|c|}{ (*) Número de Disciplinas previstas no Semestre. } & \multicolumn{2}{|c|}{ Média Geral } & $10,9 \%$ \\
\hline
\end{tabular}

Assim como na seção anterior, foi feito um cálculo para obtenção do percentual de utilização por curso através do exemplo abaixo (Tabela 05), usando os dados do Curso de Educação Física:

Tabela 05. Demonstrativo do cálculo do percentual de utilização do Moodle.

\begin{tabular}{l|c}
\hline \multicolumn{1}{c|}{ Indicador } & Total de ocorrências \\
\hline $\begin{array}{l}\text { Disciplinas Ativas do curso no Moodle } \\
\text { (Semestre 2013.2) }\end{array}$ & 11 \\
\hline $\begin{array}{l}\text { Total de Disciplinas do curso no } \\
\text { semestre Par (2013.2) }\end{array}$ & 26 \\
\hline$\%$ de utilização no Curso: & $11 / 26=0,42308=42 \%$ \\
\hline
\end{tabular}


Esses resultados evidenciam a ainda baixa utilização da plataforma virtual no auxílio ao ensino híbrido. A utilização, na maioria dos cursos, não atinge sequer os $10,9 \%$ tomados como média geral de utilização (Tabela 04). Isso significa um relevante potencial para o crescimento da modalidade na UNIVASF.

\section{Conclusões e Trabalhos Futuros}

A literatura analisada indica que o Blended Learning proporciona uma série de benefícios pedagógicos, tais como o aumento da eficácia da aprendizagem, satisfação e eficiência no processo de ensino.

Adotado na UNIVASF, como ambiente de interação aluno-professor, o Moodle, que possui funcionalidades bastante diversificadas, tem seus recursos subutilizados, tanto na sua quantidade quanto na diversidade dos seus recursos e atividades. Por outro lado, nunca houve uma ação institucional articulada para promover o uso da ferramenta de apoio ao ensino.

A utilização da Blended Learning, mesmo pequena, deve-se às iniciativas individuais dos docentes que buscam apoiar-se em instrumentos tecnológicos para auxiliar na sua atividade de ensino, já que não foi encontrado nenhum registro de iniciativa institucional para promoção do BL. Novos estudos deverão apontar possíveis razões desse baixo uso e assim subsidiar a adoção de estratégias institucionais para estimular o uso da modalidade.

Existe comprovadamente um potencial de crescimento da modalidade na UNIVASF, acompanhando inclusive seu crescimento institucional, com a implantação de novos cursos de graduação e pós-graduação que já podem iniciar suas atividades adotando a BL como abordagem pedagógica complementar. As estratégias para adoção do BL sugeridas pela literatura podem servir de referências para o um projeto institucional.

Como trabalhos futuros, a continuidade desta pesquisa também buscará evidências que expliquem o uso concentrado de determinadas funcionalidades do Moodle pelos professores e avaliará inclusive a possibilidade de adoção de outro ambiente virtual, a partir de testes de aceitação e usabilidade com usuários. A razão da existência de muitas disciplinas criadas e inativas também poderá de estudada, como forma de nortear planejamentos para a expansão da modalidade.

Serão analisados também novos relatos de experiências bem sucedidas de implantação do Blended Learning em instituições de ensino superior, para reforçar a proposta de expansão da modalidade na UNIVASF.

\section{Agradecimentos}

Os autores agradecem à UNIVASF/PRPPG, pela concessão de auxílio por meio do programa Edital Universal Mestre (18/2013) e à SEAD/UNIVASF por conceder o ambiente da pesquisa. Alex Sandro Gomes é bolsista DT Nível 2 pelo CNPq apoiado pelos processos no 310466/2012-1 e no 475634/2013-6.

\section{Referências}

Araújo, R., Panerai, T. Relato de Experiência de Blended Learning: O Moodle e o Facebook como Ambientes de Extensão da Sala de Aula Presencial. In Anais do Workshop de Informática na Escola (Vol. 1, No. 1). (2012).

Gluz, J. C., Galafassi, C., \& Penteado, F. (2011). Suporte Técnico/Pedagógico aos OAs pelos AVAs: uma Análise Comparativa das Pesquisas Recentes. XXII Simpósio Brasileiro de Informática na Educação (SBIE 2011, Aracaju-SE). 
Graham, C. R. (2013). Emerging practice and research in blended learning. In M. J. Moore (Ed.), Handbook of distance education (pp. 333 -350). (3rd ed.). New York, NY: Routledge.

Graham, C. R., Woodfield, W., \& Harrison, J. B. (2013). A framework for institutional adoption and implementation of blended learning in higher education. The Internet and Higher Education, 18, 4-14.

Halverson, L. R., Graham, C. R., Spring, K. J., \& Drysdale, J. S. (2012). An analysis of high impact scholarship and publication trends in blended learning. Distance Education, 33(3), 381 - 413.

King, S., \& Arnold, K. (2012). Blended learning environments in higher education: a case study of how professors make it happen. Mid-Western Educational Researcher, 25, 44 59.

Moskal, P., Dziuban, P., \& Hartman, J. (2013). Blended learning: a dangerous idea? The Internet and Higher Education, 18, 15 - 23.

Niemiec, M. , \& Otte, G . (2009). An administrator's guide to the whys and hows of blended learning. Journal of Asynchronous Learning Networks , 13(1), 19- 30.

Norberg, A., Dziuban, C. D., \& Moskal, P. D. (2011). A time-based blended learning model. On the Horizon, 19(3), $207-216$.

Rodrigues, L. A. (2010). Uma nova proposta para o conceito de Blended Learning. Interfaces da Educação, 1(3), 5-22.

Ross, B., \& Gage, K. (2006). Global perspectives on blended learning: Insight from WebCT and our customers in higher education. The handbook of blended learning: Global perspectives, local designs. 155-168.

Silva, J. C. S., Gomes, A. S., \& Brito, J. A. (2013). Estratégias para Blended Learning na Disciplina Resistência dos Materiais. XLI Congresso Brasileiro de Ensino em Engenharia. Gramado-RS.

Tori, R. (2009). Cursos híbridos ou blended learning. In: Litto, F. M. e Formiga, M. (Orgs.) Educação a Distância. Estado da Arte. São Paulo: Pearson, p.121-128.

Valente, J. A. (2005). Pesquisa, comunicação e aprendizagem com o computador. In: Integração das Tecnologias na Educação. Secretaria de Educação a Distância. Brasília: Ministério da Educação, Seed, p. 22-30.

Voss, G. B., Nunes, F. B., Herpich F. \& Medina, R. D. (2013). Ambientes Virtuais de Aprendizagem e Ambientes Imersivos: um estudo de caso utilizando tecnologias de computação móvel. XXIV Simpósio Brasileiro de Informática na Educação (SBIE 2013, Campinas-SP). 